Research article

Open Access

\title{
Design, Synthesis, and Antitumor Evaluation of Novel Pyrazolo[3,4-d]pyrimidine Derivatives
}

\author{
Manal M. KANDEEL, Lamia W. MoHAMED *, \\ Mohammed K. Abd El Hamid, Ahmed T. Negmeldin
}

Pharmaceutical Organic Chemistry Department, Faculty of Pharmacy, Cairo University, Cairo, Egypt.

* Corresponding author. E-mail: lamiawagdy@hotmail.com (L. W. Mohamed)

Sci Pharm. 2012; 80: 531-545

doi:10.3797/scipharm.1204-23

Published: $\quad$ June $25^{\text {th }} 2012$

Accepted: June $25^{\text {th }} 2012$

Received: $\quad$ April $23^{\text {rd }} 2012$

This article is available from: http://dx.doi.org/10.3797/scipharm.1204-23

(c) Kandeel et al.; licensee Österreichische Apotheker-Verlagsgesellschaft m. b. H., Vienna, Austria.

This is an Open Access article distributed under the terms of the Creative Commons Attribution License (http://creativecommons.org/licenses/by/3.0/), which permits unrestricted use, distribution, and reproduction in any medium, provided the original work is properly cited.

\begin{abstract}
A new series of pyrazolo[3,4- $d$ ]pyrimidines has been synthesized. The new compounds were tested for their antitumor activity on 60 different cell lines, and some of the compounds were found to have potent antitumor activity. In particular, 2-hydroxybenzaldehyde [1-(4-chlorophenyl)-3-methyl-1H-pyrazolo$[3,4-d]$ pyrimidin-4-yl]hydrazone (VIIa) was found to be the most effective among the other derivatives, showing IC50 values of 0.326 to $4.31 \mu \mathrm{M}$ on 57 different cell lines.
\end{abstract}

\section{Keywords}

Pyrazolopyrimidines • Antitumor Activity • Cytotoxic Activity • Synthesis

\section{Introduction}

Increasing interest in biological studies of pyrazolo[3,4- $d]$ pyrimidines in the last decade is a consequence of their wide usage as a pharmaceutically important class of compounds [1]. Pyrazolopyrimidine derivatives have considerable potential in the field of chemotherapy, as they were found to exhibit their antitumor activity by inhibiting different types of enzymes such as cyclin-dependent kinase [2-4], Src and Abl tyrosine kinase [5], glycogen synthase kinase-3 [6-8], adenosine deaminase [9], and epidermal growth factor receptor protein tyrosine kinase [10]. The derivatives of pyrazolo[3,4-d]pyrimidine have already been discovered as antitumor agents by the $\mathrm{NCl}$ (National Cancer Institute, USA) on HCT116 and other cell lines. The potency of these compounds is enhanced in anilide derivatives, and this translates into tumor growth inhibition in a mouse xenograft model [2]. Some pyrazolo[3,4- $d$ ]pyrimidines (1, Figure 1) structurally related with allopurinol, have also been 
reported as potent inhibitors of xanthine oxidase and the growth of several human tumor cell lines [11]. In addition, several substituted pyrazolo[3,4-d]pyrimidines (2) were reported as potent antitumor agents [12].<smiles>[R7]c1ccccc1-n1ncc2c([R])ncnc21</smiles>

1<smiles>[Al]n1ncc2c(NN=C[Te])ncnc21</smiles>

2

Fig. 1. Structures of some reported antitumor pyrazolo[3,4- $d]$ pyrimidines

Both the above findings and 4-substituted-1H-pyrazolo[3,4- $d]$-pyrimidines were reported to be cytotoxic and antitumor agents [1,13-15]. In order to explore this possibility, compounds were prepared that had diverse groups at position 4 of the pyrazolopyrimidine core, and their antitumor activity was tested.

\section{Results and Discussion}

\section{Chemistry}

The synthesis of the designed compounds is outlined in schemes 1,2 , and 3 . The main precursors for the synthesis of target derivatives, i.e. la,b and IIa-c, were prepared by a previously published synthetic method presented in Scheme 1. Compounds IIla,b were prepared according to the synthetic methods presented in Scheme 2, either by heating compounds $\mathbf{l a}, \mathbf{b}$ in formamide or by heating compounds $\mathbf{I} \mathbf{a}, \mathbf{b}$ in formic acid. Both procedures had high yields, but the second one had an even higher yield. Compound IIIc was prepared only by using one synthetic method from derivative Ilc in formic acid, as it had the better yield. Structures of newly prepared compounds were confirmed by ${ }^{1} \mathrm{H} N M R$, $\mathrm{IR}$, mass spectroscopy, and microanalyses.
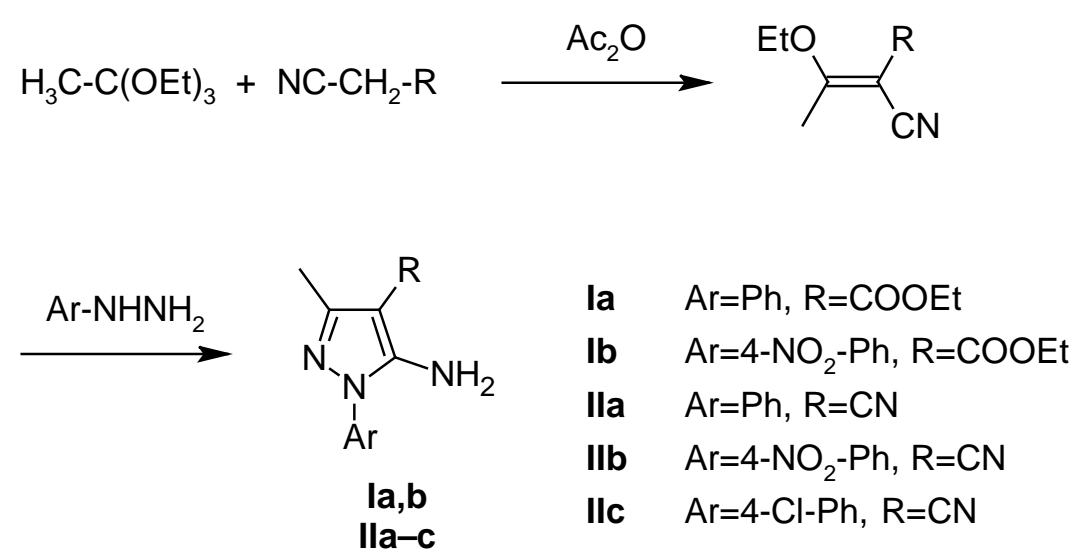

Sch. 1. Synthesis of pyrazole intermediates 
On the other hand, synthesis of IVa-c presented in scheme 2 was performed by the reflux of Illa-c in phosphorus oxychloride. The structures of the new compounds were confirmed by ${ }^{1} \mathrm{H}$ NMR, which revealed disappearance of the singlet $\mathrm{D}_{2} \mathrm{O}$ exchangeable signal corresponding to $\mathrm{NH}$, and showed an increased deshielding of $\mathrm{H}$ at position 6 , due to the inductive effect of the chlorine atom. It was also confirmed by mass spectra which gave fragments showing the isotopic pattern of the chlorine atom.<smiles>CCOC(=O)c1c(C)nn(C)c1N</smiles>

la,b<smiles>Cc1nn([Ga])c(N)c1C#N</smiles>

IIa-c<smiles>Cc1nn(C)c2nc[nH]c(=O)c12</smiles>

IIIa-c<smiles>Cc1nn([Al])c2ncnc(Cl)c12</smiles>

IVa-c $\mathrm{HCOOH}$

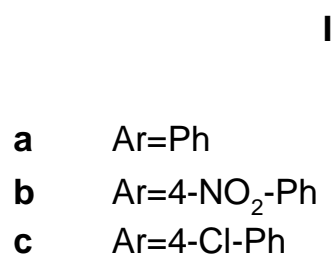

c $\mathrm{Ar}=4-\mathrm{Cl}-\mathrm{Ph}$

Sch. 2. Synthesis of pyrazolo[3,4-d]pyrimidines

The synthesis of Va-g and VIla-c was outlined in scheme 3. First, Va-g were obtained by the reflux of IVa-c with the appropriate amine using triethylamine as a catalyst, and the formed derivatives were confirmed by ${ }^{1} \mathrm{H}$ NMR, which revealed appearance of the singlet $\mathrm{D}_{2} \mathrm{O}$ exchangeable signal corresponding to $\mathrm{NH}$, and appearance of other signals characterizing the introduced groups. The structures of some of these derivatives were additionally confirmed by mass spectra. ${ }^{13} \mathrm{C}$ NMR was performed on compounds Va, Vc, and VIla. Va showed distinct aromatic carbons where the carbon at position 3 of the pyrazole ring appeared at 101.76, while that of the pyrimidine ring at position 6 was the highest desheilded and appeared at $156.35 \mathrm{ppm}$. On the other hand, the aromatic ring at position 1 of the pyrazole ring gives aromatic carbons, which are given the numbers $1^{\prime}, 2^{\prime}, 3^{\prime}, 4^{\prime}, 5^{\prime}$, and $6^{\prime}$, and that attached to the amine at the pyrimidine ring gives aromatic carbons marked as 1", 2", 3", 4", 5" and 6". Vc showed two distinct doublet signals belonging to the two $\mathrm{CH}_{2}$ groups attached to the $\mathrm{NH}$ group. The presence of the ethylene spacer was further confirmed by DEPT technique.

Second, VIla-c were synthesized by the reflux of IVc with hydrazine, producing VI which was confirmed by ${ }^{1} \mathrm{H}$ NMR, IR spectrum, microanalysis, and mass spectrum in which appearance of a singlet proton at $\delta 8.36 \mathrm{ppm}$ in ${ }^{1} \mathrm{H}$ NMR indicated slight shielding of the proton at position 6 of the pyrimidine ring, due to the removal of the chlorine atom, and a broad range of the $\mathrm{D}_{2} \mathrm{O}$ exchangeable signal of $\mathrm{NH}$ and $\mathrm{NH}_{2}$ groups at 3.82 and 4.95 ppm, and by the mass spectrum which shows $\mathrm{M}^{+}, \mathrm{M}^{+}+2$ at $\mathrm{m} / \mathrm{z} 274,276$ as $100.00 \%, 49.15 \%$.

Furthermore, VI was condensed with appropriate aldehydes producing VIla-c, the structure of which was confirmed by ${ }^{1} \mathrm{H}$ NMR, ${ }^{13} \mathrm{C}$ NMR, IR spectra, microanalyses, and 
mass spectra. The IR spectrum shows the disappearance of the IR peak equivalent to $\mathrm{NH}_{2}$. In ${ }^{1} \mathrm{H}$ NMR, the appearance of the $\mathrm{D}_{2} \mathrm{O}$ exchangeable broad singlet indicated the presence of $\mathrm{NH}$, deshielded singlet of $\mathrm{N}=\mathrm{CH}$, in addition to different peaks equivalent to different substituents in each derivative. The $\mathrm{OH}$ group of compound VIla appeared in the IR at $3369 \mathrm{~cm}^{-1}$ as an exchangeable peak in ${ }^{1} \mathrm{H}$ NMR at10.20ppm. The $\mathrm{OCH}_{3}$ group of compound VIIb was detected by ${ }^{1} \mathrm{H}$ NMR appearing more deshielded at $3.82 \mathrm{ppm}$ than the $\mathrm{CH}_{3}$ group at position 3 at $2.79 \mathrm{ppm}$. Compound VIIc mainly showed the $\mathrm{NO}_{2}$ group in the IR at 1437, 1346 and the aromatic protons of the benzene ring 2", 4", 5", and 6" with a deshielded singlet signal equivalent to $2 "$ at 8.7-8.8, proving the m-substitution.<smiles>Cc1nn([Al])c2ncnc(Cl)c12</smiles>

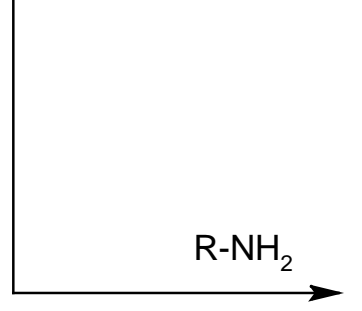<smiles>Cc1nn(-c2ccc(Cl)cc2)c2ncnc(N)c12</smiles>

VI<smiles>[R]Nc1ncnc2c1c(C)nn2[Al]</smiles>

Va-g<smiles>[R]C=C(C)O</smiles><smiles>[R]C=NNc1ncnc2c1c(C)nn2-c1ccc(Cl)cc1</smiles>

VIIa-c

$$
\begin{array}{ll}
\text { Va } & \mathrm{Ar}=\mathrm{R}=\mathrm{Ph} \\
\text { Vb } & \mathrm{Ar}=\mathrm{Ph}, \mathrm{R}=4-\mathrm{OCH}_{3}-\mathrm{Ph} \\
\text { Vc } & \mathrm{Ar}=\mathrm{Ph}, \mathrm{R}=2-\mathrm{Ph}-\mathrm{C}_{2} \mathrm{H}_{4} \\
\text { Vd } & \mathrm{Ar}=4-\mathrm{NO}_{2}-\mathrm{Ph}, \mathrm{R}=\mathrm{Ph} \\
\text { Ve } & \mathrm{Ar}=4-\mathrm{NO}_{2}-\mathrm{Ph}, \mathrm{R}=2-\mathrm{Ph}-\mathrm{C}_{2} \mathrm{H}_{4} \\
\text { Vf } & \mathrm{Ar}=4-\mathrm{Cl}-\mathrm{Ph}, \mathrm{R}=4-\mathrm{OC} \mathrm{H}_{3}-\mathrm{Ph} \\
\text { Vg } & \mathrm{Ar}=4-\mathrm{Cl}-\mathrm{Ph}, \mathrm{R}=2-\mathrm{Ph}-\mathrm{C}_{2} \mathrm{H}_{4} \\
\text { VIla } & \mathrm{R}=2-\mathrm{OH}-\mathrm{Ph} \\
\text { VIIb } & \mathrm{R}=4-\mathrm{OCH}_{3}-\mathrm{Ph} \\
\text { VIIc } & \mathrm{R}=3-\mathrm{NO}_{2}-\mathrm{Ph}
\end{array}
$$

Sch. 3. Synthesis of new pyrazolo[3,4-d]pyrimidine derivatives

\section{Antitumor activity}

The antitumor activity was determined for the newly synthesized compounds at the $\mathrm{NCl}$ for in vitro one-dose testing and detection of IC50 of their antitumor activity on 60 different cell lines. Compound Vc, Vg, VIla, and VIIc were found to have the highest inhibitory activity on many cell lines. The obtained results of the tested derivatives showed a distinctive potential pattern of selectivity, as well as broad-spectrum antitumor activity (Table 1). Compound VIla was subjected to 5-dose testing, as it showed the highest activity among other derivatives showing inhibition of 57 different cell lines (Table 2). 
Tab. 1. Inhibition percent of the tested compounds $\left(10^{-5}\right.$ Molar) on different 60 cell lines

\begin{tabular}{|c|c|c|c|c|c|c|c|c|c|c|c|}
\hline \multirow{2}{*}{\multicolumn{2}{|c|}{ Cell line type }} & \multicolumn{10}{|c|}{ Tested compounds and inhibition percent of cell lines } \\
\hline & & Va & Vb & Vc & Vd & Ve & Vf & Vg & VIIa & VIIb & VIIC \\
\hline \multirow{6}{*}{ 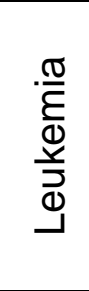 } & CCRF-CEM & 19.43 & 4.00 & 6.94 & 2.25 & - & 2.45 & 0.33 & 93.59 & - & 79.59 \\
\hline & HL-60(TB) & 29.20 & 11.40 & 22.22 & 8.87 & 35.22 & - & 29.98 & 73.60 & - & 69.05 \\
\hline & K-562 & 30.71 & 24.64 & 65.57 & 5.24 & 23.27 & - & 76.32 & 81.45 & 19.22 & 82.62 \\
\hline & MOLT-4 & 30.65 & 32.39 & 53.25 & 21.34 & 35.41 & 12.37 & 43.41 & 90.57 & 20.53 & 84.60 \\
\hline & RPMI-8226 & 23.57 & 9.63 & 12.33 & 16.39 & 3.17 & 19.90 & 17.27 & 62.86 & 6.07 & 33.66 \\
\hline & SR & 36.93 & 41.72 & 53.71 & 28.86 & 37.77 & 20.94 & 34.91 & 95.20 & 18.44 & 48.73 \\
\hline \multirow{9}{*}{ 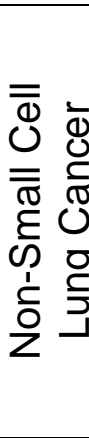 } & A549/ATCC & - & 4.05 & 6.18 & 10.26 & 0.26 & 3.61 & - & 90.54 & - & - \\
\hline & EKVX & - & 5.68 & 8.75 & - & 47.45 & 13.84 & 19.40 & 61.94 & - & 15.99 \\
\hline & HOP-62 & - & 2.91 & 9.60 & - & - & 19.97 & 7.30 & 92.07 & - & - \\
\hline & HOP-92 & 34.14 & 8.10 & 11.46 & 6.51 & 8.78 & 28.98 & 51.17 & 55.93 & 12.52 & 79.55 \\
\hline & $\mathrm{NCl}-\mathrm{H} 226$ & - & - & - & - & 2.27 & 10.33 & 3.81 & 47.25 & - & - \\
\hline & $\mathrm{NCl}-\mathrm{H} 23$ & - & - & - & - & 4.22 & 10.44 & 4.32 & 63.00 & - & 5.70 \\
\hline & $\mathrm{NCl}-\mathrm{H} 322 \mathrm{M}$ & - & - & - & - & - & & - & 66.66 & - & 3.60 \\
\hline & $\mathrm{NCl}-\mathrm{H} 460$ & - & - & - & 10.24 & 8.52 & $4 . \overline{2} 0$ & 3.49 & 94.96 & - & 22.17 \\
\hline & $\mathrm{NCl}-\mathrm{H}$ & 8.39 & 9.21 & 17.72 & 19.25 & - & 28.80 & 4.48 & & - & - \\
\hline \multirow{7}{*}{ 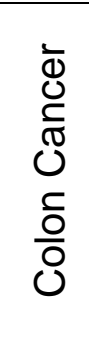 } & COLO 205 & 4.67 & - & - & - & - & 8.75 & 6.22 & 81.33 & - & 4.75 \\
\hline & HCC-2998 & - & - & - & - & - & - & & 68.95 & - & 5.17 \\
\hline & НСТ-116 & - & 0.89 & 6.87 & 33.53 & 7.57 & 8.26 & $5 . \overline{9} 3$ & 73.26 & - & - \\
\hline & НСТ-15 & 3.74 & 9.57 & 7.60 & - & 4.73 & 6.88 & 6.10 & 68.08 & - & - \\
\hline & HT29 & 12.25 & 18.87 & 5.24 & 10.25 & - & 14.96 & 4.20 & 79.92 & 0.97 & 9.36 \\
\hline & KM12 & - & - & - & - & 4.92 & 5.48 & - & 71.56 & - & 33.45 \\
\hline & SW-620 & 0.79 & - & - & - & 0.72 & 2.59 & - & 64.89 & - & 11.24 \\
\hline \multirow{6}{*}{ 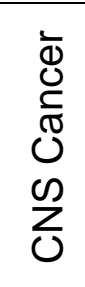 } & SF-268 & 5.96 & - & - & 3.91 & 6.41 & - & - & 90.33 & - & 41.99 \\
\hline & SF-295 & - & - & 2.42 & - & - & - & - & 75.51 & - & - \\
\hline & SF-539 & 3.88 & 0.50 & - & 1.85 & 0.58 & 9.76 & - & 85.97 & - & - \\
\hline & SNB-19 & - & - & - & 2.95 & 2.93 & 1.78 & - & 70.42 & - & 3.66 \\
\hline & SNB-75 & 0.60 & 10.60 & - & - & 12,15 & 10.17 & - & 36.41 & 0.88 & 0.87 \\
\hline & U251 & - & - & - & 0.50 & - & - & - & 86.92 & 0.73 & 17.78 \\
\hline \multirow{8}{*}{$\begin{array}{l}\frac{\sigma}{E} \\
\frac{0}{0} \\
\frac{\sigma}{\sigma} \\
\frac{\mathbb{d}}{2}\end{array}$} & LOX IMVI & - & 1.66 & 7.32 & 15.16 & - & 19.19 & - & 64.88 & 0.69 & 6.68 \\
\hline & MALME-3M & - & - & - & - & - & & - & 51.30 & - & 2.87 \\
\hline & MDAMB435 & - & - & 6.98 & - & 5.22 & $\overline{-}$ & - & 72.73 & - & 2.94 \\
\hline & SK-MEL-2 & - & - & - & - & - & 10.73 & 5.94 & 87.67 & 0.78 & 1.14 \\
\hline & SK-MEL-28 & - & - & - & - & - & - & - & 74.82 & - & - \\
\hline & SK-MEL-5 & - & - & 2.47 & - & - & 3.25 & - & 16.17 & - & - \\
\hline & UACC-257 & - & - & - & - & - & - & - & 74.83 & - & - \\
\hline & UACC-62 & - & 1.44 & 10.31 & 0.78 & 9.62 & 18.24 & 14.27 & 86.54 & - & - \\
\hline
\end{tabular}


Tab. 1. (Cont.)

\begin{tabular}{|c|c|c|c|c|c|c|c|c|c|c|c|}
\hline \multirow{2}{*}{\multicolumn{2}{|c|}{ Cell line type }} & \multicolumn{10}{|c|}{ Tested compounds and inhibition percent of cell lines } \\
\hline & & Va & Vb & Vc & Vd & Ve & Vf & Vg & VIIa & VIIb & VIIC \\
\hline \multirow{7}{*}{ 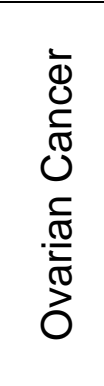 } & IGROV1 & - & - & 6.94 & - & 0.87 & & - & 76.08 & - & 5.25 \\
\hline & OVCAR-3 & - & - & - & - & - & $\overline{-}$ & - & 65.08 & - & 91.83 \\
\hline & OVCAR-4 & - & 2.38 & 1.30 & - & - & 8.86 & - & 80.50 & - & 5.56 \\
\hline & OVCAR-5 & 5.33 & 15.68 & 8.84 & - & - & 4.24 & 7.88 & 52.48 & - & - \\
\hline & OVCAR-8 & - & 3.30 & 1.97 & 0.91 & 1.68 & 6.25 & - & 85.94 & - & 0.43 \\
\hline & $\begin{array}{l}\text { NCl/ADR- } \\
\text { RES }\end{array}$ & - & 7.90 & 21.95 & - & - & - & 5.66 & 80.90 & - & - \\
\hline & SK-OV-3 & - & - & - & - & - & 24.57 & 7.19 & 95.56 & - & - \\
\hline \multirow{8}{*}{ 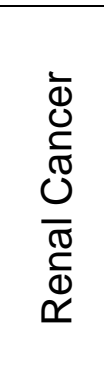 } & 786 & 3.00 & 8.93 & 11.46 & - & - & - & - & 91.01 & - & 3.71 \\
\hline & A498 & 18.12 & 12.14 & 7.44 & - & 18.37 & - & - & 21.46 & 8.64 & 14.62 \\
\hline & $\mathrm{ACHN}$ & 0.89 & 6.27 & 5.86 & - & 0.28 & 13.55 & 7.13 & 83.54 & - & - \\
\hline & CAKI-1 & - & - & 23.73 & - & 12.87 & - & 29.75 & 86.47 & 4.83 & 24.71 \\
\hline & RXF 393 & - & - & - & - & - & 2.16 & 2.27 & 74.27 & - & - \\
\hline & SN12C & - & - & - & 1.51 & - & 7.73 & - & 76.94 & - & - \\
\hline & TK-10 & - & 5.94 & 5.32 & 70.00 & - & 16.23 & 5.88 & 83.60 & 2.97 & 6.83 \\
\hline & UO-31 & - & 4.94 & 12.46 & 0.50 & 20.39 & - & 13.47 & 64.77 & 9.87 & 27.79 \\
\hline Prost. & PC-3 & 6.67 & 7.99 & 1.59 & 8.91 & 11.43 & 24.61 & 22.41 & 58.41 & 10.93 & 61.01 \\
\hline & DU-145 & - & - & - & - & - & - & - & 80.91 & - & 31.32 \\
\hline \multirow{6}{*}{ 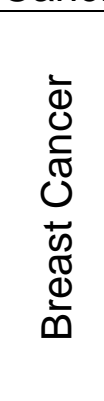 } & MCF7 & - & 4.97 & - & 6.68 & 6.98 & 15.10 & 1.80 & 91.72 & 4.60 & - \\
\hline & $\begin{array}{l}\text { MDA-MB- } \\
\text { 231/ATCC }\end{array}$ & - & - & - & 4.83 & - & 24.17 & 14.77 & 67.43 & 2.75 & - \\
\hline & HS578T & - & - & - & - & - & - & - & 19.00 & 0.98 & 0.81 \\
\hline & ВТ-549 & - & - & - & - & - & - & - & 63.98 & - & - \\
\hline & T-47D & - & - & 12.42 & 3.82 & 6.98 & 23.74 & 2.13 & 86.73 & - & - \\
\hline & $\begin{array}{l}\text { MDA-MB- } \\
468\end{array}$ & - & - & - & - & - & - & - & 47.74 & - & - \\
\hline
\end{tabular}

\section{Experimental}

\section{Chemistry}

All melting points were determined on the Stuart apparatus and the values given are uncorrected. The IR spectra were determined on the Shimadzu IR 435 spectrophotometer $\left(\mathrm{KBr}, \mathrm{cm}^{-1}\right)$, Faculty of Pharmacy, Cairo University, Egypt. The ${ }^{1} \mathrm{H} N M R$ and ${ }^{13} \mathrm{C} N M R$ spectra were recorded on the Varian Gemini $75 \mathrm{MHz}$ spectrophotometer using TMS as the internal standard. Chemical shift values are recorded in ppm on the $\delta$ scale, Microanalysis Center, Cairo University, Egypt. Mass spectra were recorded on a Hewlett Packard 5988 spectrometer, Microanalysis Center, Cairo University, Egypt. Elemental analyses were carried out at the Microanalysis Center, Cairo University, Egypt; the values found were within $\pm 0.35 \%$ of the theoretical ones. Progress of the reactions was monitored using TLC sheets precoated with the UV fluorescent silica gel, Merck 60F 254, and were visualized using a UV lamp. 
Tab. 2. Values detected from 5 dilutions for compound (VIla) on 60 cell lines

\begin{tabular}{|c|c|c|c|c|c|c|c|c|c|}
\hline \multicolumn{2}{|c|}{ Cell line type } & $\begin{array}{l}\text { GI50 } \\
(\mu M)\end{array}$ & $\begin{array}{l}\text { TGI } \\
(\mu \mathrm{M})\end{array}$ & $\begin{array}{l}\text { LC50 } \\
(\mu \mathrm{M})\end{array}$ & \multicolumn{2}{|c|}{ Cell line type } & $\begin{array}{l}\text { GI50 } \\
(\mu M)\end{array}$ & $\begin{array}{l}\text { TGI } \\
(\mu \mathrm{M})\end{array}$ & $\begin{array}{l}\text { LC50 } \\
(\mu \mathrm{M})\end{array}$ \\
\hline \multirow{6}{*}{ 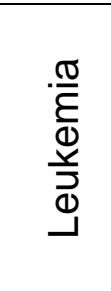 } & CCRF-CEM & 0.326 & - & $>100$ & \multirow{7}{*}{ 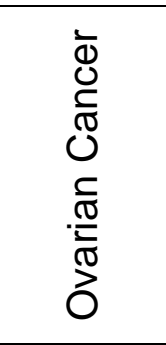 } & & 0.546 & 10.7 & $>100$ \\
\hline & & 0.522 & 48.6 & $>100$ & & OVCA & 0.427 & 23.5 & 97.1 \\
\hline & & 1.37 & $>100$ & $>100$ & & OVCAR-4 & 0.578 & 73.4 & $>100$ \\
\hline & MOLT. & 0.625 & $>100$ & $>100$ & & OVCAR-5 & 3.66 & $>100$ & $>100$ \\
\hline & RPN & 0.760 & $>100$ & $>100$ & & OVC & 0.477 & 36.1 & $>100$ \\
\hline & SR & 0.663 & 53.4 & $>100$ & & R-RES & 0.419 & 22.6 & $>100$ \\
\hline \multirow{9}{*}{ 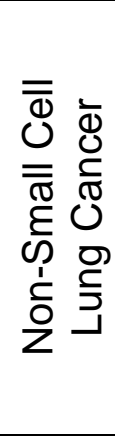 } & 49/ATCC & 2.22 & 17.8 & 95.7 & & & 0.601 & 37.4 & $>100$ \\
\hline & & 1.50 & 52.0 & $>100$ & \multirow{8}{*}{ 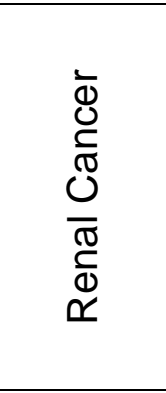 } & & 1.58 & 15.8 & $>100$ \\
\hline & $\mathrm{HC}$ & 0.559 & 24.0 & $>100$ & & & 17.0 & 68.3 & $>100$ \\
\hline & $\mathrm{HC}$ & 0.383 & 30.9 & $>100$ & & & 0.350 & 10.8 & 92.9 \\
\hline & $\mathrm{NCl}$ & 4.23 & 62.9 & $>100$ & & & 0.360 & 10.5 & $>100$ \\
\hline & $\mathrm{NCl}-\mathrm{I}$ & 2.97 & $>100$ & $>100$ & & $\mathrm{RX}$ & 4.31 & 30.7 & $>100$ \\
\hline & $\mathrm{NC}$ & 0.335 & 22.0 & $>100$ & & & .983 & 84.0 & $>100$ \\
\hline & & 0.398 & 3.61 & 45.0 & & & 2.60 & 46.9 & $>100$ \\
\hline & & 1.79 & $>100$ & $>100$ & & & 0.348 & 3.51 & $>100$ \\
\hline \multirow{6}{*}{ 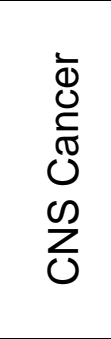 } & & 0.646 & 23.0 & $>100$ & & & 1.36 & 92.3 & $>100$ \\
\hline & & 1.67 & 20.0 & $>100$ & & & 0.601 & 16.7 & $>100$ \\
\hline & & 0.522 & 8.84 & 96.9 & \multirow{6}{*}{ 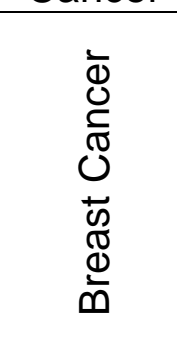 } & & 1.56 & $>100$ & $>100$ \\
\hline & SNB-19 & 1.55 & 64.6 & $>100$ & & $\begin{array}{l}\text { MDA-MB-231 } \\
\text { /ATCC }\end{array}$ & 0.719 & 42.7 & $>100$ \\
\hline & & 8.66 & 53.0 & $>100$ & & & 7.34 & 77.6 & $>100$ \\
\hline & & 0.595 & 16.9 & 95.1 & & & 3.05 & 78.3 & $>100$ \\
\hline \multirow{9}{*}{$\begin{array}{l}\frac{\pi}{\tilde{\sigma}} \\
\frac{0}{\sigma} \\
\frac{\sigma}{0} \\
\sum\end{array}$} & LOX IMVI & 0.389 & 11.9 & 57.1 & & & 2.58 & $>100$ & $>100$ \\
\hline & MALME-3M & 1.19 & 9.56 & $>100$ & & & 2.15 & 7.81 & $>100$ \\
\hline & M14 & 0.726 & 2.68 & 8.83 & \multirow{7}{*}{ 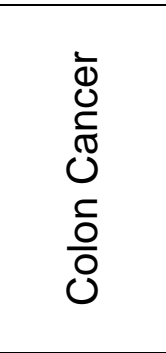 } & $\mathrm{CO}$ & 1.27 & $>100$ & $>100$ \\
\hline & MDAMB435 & 1.25 & $>100$ & $>100$ & & HCC-2998 & 0.656 & $>100$ & $>100$ \\
\hline & SK-ME & 2.97 & 19.0 & $>100$ & & НСТ-116 & 0.373 & 24.9 & $>100$ \\
\hline & SK-M & 3.70 & $>100$ & $>100$ & & НСТ-15 & 0.409 & $>100$ & $>100$ \\
\hline & & 1.06 & 2.55 & 6.11 & & НT29 & 2.94 & $>100$ & $>100$ \\
\hline & & 3.49 & 24.7 & $>100$ & & KM12 & 0.686 & $>100$ & $>100$ \\
\hline & UACC-62 & 1.75 & 7.78 & $>100$ & & SW-620 & 2.31 & $>100$ & $>100$ \\
\hline
\end{tabular}

The synthesis of the target compounds is outlined in Schemes 1-3. Compounds la [16], Ib [17], Ila-c [18-20], IIla [21], and Iva [22] were prepared as reported in the literature.

\section{Procedures for the synthesis of compounds IIIb and IIIC}

Method 1

A suspension of the appropriate derivative la,b $(0.01 \mathrm{~mol})$ in formamide $(30 \mathrm{ml})$ was stirred at $145^{\circ} \mathrm{C}$ for $3 \mathrm{~h}$; the solution was then cooled by being poured on ice-cold water, filtered, washed with water, dried, and finally crystallized from formic acid. 


\section{Method 2}

A suspension of the appropriate derivative Ila-c $(0.01 \mathrm{~mol})$ in $85 \%$ formic acid $(40 \mathrm{ml})$, was heated under reflux for $7 \mathrm{~h}$; the reaction mixture was then cooled, filtered, washed with water, dried, and crystallized from formic acid.

\section{3-Methyl-1-(4-nitrophenyl)-1,5-dihydro-4H-pyrazolo[3,4-d]pyrimidin-4-one (IIIb)}

Yield: $2.6 \mathrm{~g}\left(96 \%\right.$ by method 2); M.p.: $>300^{\circ} \mathrm{C} ;{ }^{1} \mathrm{H}$ NMR $(300 \mathrm{MHz}$, DMSO-d 6 ): $\delta=3.15$ (s, $\left.3 \mathrm{H}, \mathrm{CH}_{3}\right) ; 8.20(\mathrm{~s}, 1 \mathrm{H}, \mathrm{C} 6-\mathrm{H}) ; 8.34(\mathrm{~d}, \mathrm{~J}=9.3 \mathrm{~Hz}, 2 \mathrm{H}$, ArH C2',6'); 8.39 (d, J = 9.6 Hz, $2 \mathrm{H}$, ArH C3',5'); 12.51 (s, 1H, NH, D $\mathrm{O}$ exchangeable) ppm; IR ( $\left.\mathrm{cm}^{-1}\right)$ : 3370(NH), 3075, 3034 ( $\mathrm{CH}$ aromatic), 2911 ( $\mathrm{CH}$ aliphatic), $1686(\mathrm{C}=\mathrm{O}), 1589(\mathrm{C}=\mathrm{N}), 1441,1337\left(\mathrm{NO}_{2}\right) ; \mathrm{MS}(70 \mathrm{ev})$ : $\mathrm{m} / \mathrm{z} 271\left(\mathrm{M}^{+}, 100 \%\right)$. Anal. Calcd for $\mathrm{C}_{12} \mathrm{H}_{9} \mathrm{~N}_{5} \mathrm{O}_{3}$ (271.23): C, 53.14; $\mathrm{H}, 3.34 ; \mathrm{N}, 25.82$. Found: C, 53.30; H, 3.64; N, 26.36

\section{1-(4-Chlorophenyl)-3-methyl-1,5-dihydro-4H-pyrazolo[3,4-d]pyrimidin-4-one (IIIc)}

Yield: $1.94 \mathrm{~g}\left(74 \%\right.$ by method 2); M.p.: $>300^{\circ} \mathrm{C} ;{ }^{1} \mathrm{H}$ NMR $\left(300 \mathrm{MHz}, \mathrm{DMSO}-\mathrm{d}_{6}\right): \delta=3.30$ (s, 3H, $\mathrm{CH}_{3}$ ); 7.60 (d, J = 9.0 Hz, 2H, ArH C3',5'); 8.08 (d, 2H, J = 9.0 Hz, ArH C2',6'); 8.15 (s, 1H, C6-H); 12.37 (s, 1H, NH, D2O exchangeable) ppm; IR $\left(\mathrm{cm}^{-1}\right): 3422(\mathrm{NH}), 3121$, 3040 ( $\mathrm{CH}$ aromatic) 2974 ( $\mathrm{CH}$ aliphatic),1670 (C=O), $1589(\mathrm{C}=\mathrm{N}) ; \mathrm{MS}(70 \mathrm{ev}): \mathrm{m} / \mathrm{z} 261$ $\left(\mathrm{M}^{+}+1,9.24 \%\right)$. Anal. Calcd for $\mathrm{C}_{12} \mathrm{H}_{9} \mathrm{ClN}_{4} \mathrm{O}$ (260.68): $\mathrm{C}, 55.29 ; \mathrm{H}, 3.48 ; \mathrm{N}, 21.49$. Found: C, 55.36; H, 3.69; N, 21.41.

\section{General procedure for the synthesis of compounds IVb and IVc}

A suspension of the appropriate derivative Illa-c $(0.01 \mathrm{~mol})$ in phosphorus oxychloride $(80 \mathrm{ml})$ was heated under reflux for $3 \mathrm{~h}$; the solution was cooled and then poured onto icecold water. The precipitated product was filtered, dried, and crystallized from ethanol.

4-Chloro-3-methyl-1-(4-nitrophenyl)-1H-pyrazolo[3,4-d]pyrimidine (IVb)

Yield: $2.03 \mathrm{~g}(70 \%)$, M.p.: $210-212^{\circ} \mathrm{C} ;{ }^{1} \mathrm{H}$ NMR $\left(200 \mathrm{MHz}, \mathrm{DMSO}-\mathrm{d}_{6}\right): \delta=2.71(\mathrm{~s}, 3 \mathrm{H}$, $\mathrm{CH}_{3}$ ); 8.37 (d, $\left.\mathrm{J}=9.4 \mathrm{~Hz}, 2 \mathrm{H}, \mathrm{ArH} \mathrm{C2}, 6^{\prime}\right) ; 8.41$ (d, $\mathrm{J}=9.4 \mathrm{~Hz}, 2 \mathrm{H}$, ArH C3',5'); 8.96 (s, $1 \mathrm{H}$, C6-H) ppm; IR (cm $\left.{ }^{-1}\right)$ : 3120, 3080 (CH aromatic), 2905 ( $\mathrm{CH}$ aliphatic), 1597,1576 (C=N), 1445, $1341\left(\mathrm{NO}_{2}\right)$; MS (70 ev): $\mathrm{m} / \mathrm{z} 289\left(\mathrm{M}^{+}, 100 \%\right)$. Anal. Calcd for $\mathrm{C}_{12} \mathrm{H}_{8} \mathrm{ClN}_{5} \mathrm{O}_{2}$ (289.68): C, 49.75; H, 2.78; N, 24.18. Found: C, 49.60; H, 2.90; N, 24.22.

\section{4-Chloro-1-(4-chlorophenyl)-3-methyl-1H-pyrazolo[3,4-d]pyrimidine (IVc)}

Yield: $1.48 \mathrm{~g}(53 \%)$, M.p.: $189-190^{\circ} \mathrm{C} ;{ }^{1} \mathrm{H}$ NMR (300 MHz, DMSO-d 6 ): $\delta=3.40(\mathrm{~s}, 3 \mathrm{H}$, $\mathrm{CH}_{3}$ ); 7.65 (d, J = 9.0 Hz, 2H, ArH C3',5'); 8.16 (d, 2H, J = 9.0 Hz, ArH C2',6'); 8.92 (s, 1H, C6-H) ppm; IR (cm $\left.{ }^{-1}\right):$ 3095, 3065 (CH aromatic), 2924 (CH aliphatic), 1589,1574 (C= N); MS (70 ev): $\mathrm{m} / \mathrm{z} 278\left(\mathrm{M}^{+}, 87.22\right)$. Anal. Calcd for $\mathrm{C}_{12} \mathrm{H}_{8} \mathrm{Cl}_{2} \mathrm{~N}_{4}$ (279.12): $\mathrm{C}, 51.64 ; \mathrm{H}, 2.89$; N, 20.07. Found: C, 51.43; H, 3.01; N, 19.81.

\section{General procedure for the synthesis of compounds Va-g}

A suspension of the appropriate derivative IVa-c $(0.01 \mathrm{~mol})$ and the appropriate amine $(0.01 \mathrm{~mol})$ in ethanol $(30 \mathrm{ml})$, triethylamine $(0.3 \mathrm{~g}, 0.03 \mathrm{~mol})$, was added and the reaction mixture was heated under reflux for $2-7 \mathrm{~h}$; (the reaction was monitored using TLC until the starting materials were consumed in the reaction). The reaction mixture was allowed to 
cool leading to separation of the product, and then the crude product was filtered, dried, and crystallized from the appropriate solvent.

\section{3-Methyl-N,1-diphenyl-1H-pyrazolo[3,4-d]pyrimidin-4-amine (Va)}

Yield: $0.15 \mathrm{~g}$ (49\%), M.p.: $144-145^{\circ} \mathrm{C}$ (ethanol/water); ${ }^{1} \mathrm{H}$ NMR $\left(200 \mathrm{MHz}, \mathrm{CDCl}_{3}\right): \delta=$ $2.88\left(\mathrm{~s}, 3 \mathrm{H}, \mathrm{CH}_{3}\right) ; 7.00(\mathrm{~s}, 1 \mathrm{H}, \mathrm{NH}) ; 7.20-7.35$ (m, 3H, ArH C3",4",5"); 7.40-7.55 (m, 3H, ArH C3',4',5'); 7.72 (d, J = 9.0 Hz, 2H, ArH C2",6"); 8.20 (d, J = 9.0 Hz, 2H, ArH C2',6'); $8.55(\mathrm{~s}, 1 \mathrm{H}, \mathrm{C} 6-\mathrm{H})$ ppm.; ${ }^{13} \mathrm{C}$ NMR $\left(75 \mathrm{MHz}, \mathrm{CDCl}_{3}\right): \delta=15.17$ (q, 1C, $\left.\mathrm{CH}_{3}\right) ; 101.76(\mathrm{~s}$, 1C, C 3a); 121.48 (d, 2C, ArC 2",6"); 121.82 (d, 2C, ArC 2',6'); 124.84 (d, 1C, ArC 4"); 126.38 (d, 1C, ArC 4'); 129.15 (d, 2C, ArC 3',5'); 129.22 (d, 2C, ArC 3",5"); 137.73 (s, 1C, ArC 1'); 138.73 (s, 1C, ArC 1"); 140.73 (s, 1C, C 3); 154.30 (s, 1C, C 7a); 155.60 (s, 1C, C 4); 156.35 (d, 1C, C 6) ppm; IR (cm $\left.{ }^{-1}\right)$ : $3455(\mathrm{NH}), 3080,3020(\mathrm{CH}$ aromatic), $2930(\mathrm{CH}$ aliphatic); MS (70 ev): $\mathrm{m} / z 301\left(\mathrm{M}^{+}, 78.78 \%\right)$. Anal. Calcd for $\mathrm{C}_{18} \mathrm{H}_{15} \mathrm{~N}_{5}$ (301.35): C, 71.74; H, 5.02; N, 23.24. Found: C, 72.00; H, 4.97; N, 23.07.

\section{N-(4-Methoxyphenyl)-3-methyl-1-phenyl-1H-pyrazolo[3,4-d]pyrimidin-4-amine (Vb)}

Yield: $0.20 \mathrm{~g}(59 \%)$, M.p.: $121-122^{\circ} \mathrm{C}$ (ethanol/water); ${ }^{1} \mathrm{H}$ NMR $\left(200 \mathrm{MHz}\right.$, DMSO- $\left.\mathrm{d}_{6}\right): \delta=$ $2.77\left(\mathrm{~s}, 3 \mathrm{H}, \mathrm{CH}_{3}\right) ; 3.80\left(\mathrm{~s}, 3 \mathrm{H}, \mathrm{OCH}_{3}\right) ; 6.99$ (d, J = 6.6 Hz, 2H, ArH C3",5"); 7.32 (t, J = 6.6 $\mathrm{Hz}, 1 \mathrm{H}$, ArH C4'); 7.55 (d, $\mathrm{J}=6.0 \mathrm{~Hz} \& \mathrm{~m}, 4 \mathrm{H}$, ArH C2",6", 3', 5'); 8.20 (d, $\mathrm{J}=8.2 \mathrm{~Hz}, 2 \mathrm{H}$, ArH C2',6'); 8.38 (s, 1H, C6-H); 8.76 (s, 1H, NH, D2O exchangeable) ppm; IR (cm $\left.{ }^{-1}\right): 3439$ $(\mathrm{NH}), 3080,3028$ ( $\mathrm{CH}$ aromatic), 2976 ( $\mathrm{CH}$ aliphatic). Anal. Calcd for $\mathrm{C}_{19} \mathrm{H}_{17} \mathrm{~N}_{5} \mathrm{O}$ (331.37): C, 68.87; H, 5.17; N, 21.13. Found: C, 68.90; H, 5.00; N, 21.01

\section{3-Methyl-1-phenyl-N-(2-phenylethyl)-1H-pyrazolo[3,4-d]pyrimidin-4-amine (Vc)}

Yield: $0.10 \mathrm{~g}(30 \%)$, M.p.: $151-152^{\circ} \mathrm{C}$ (ethanol/water); ${ }^{1} \mathrm{H}$ NMR $\left(200 \mathrm{MHz}, \mathrm{CDCl}_{3}\right): \delta=$ $2.45\left(\mathrm{~s}, 3 \mathrm{H}, \mathrm{CH}_{3}\right) ; 3.05\left(\mathrm{t}, \mathrm{J}=9.0 \mathrm{~Hz}, 2 \mathrm{H}, \mathrm{CH}_{2}\right) ; 3.88\left(\mathrm{t}, \mathrm{J}=9.0 \mathrm{~Hz}, 2 \mathrm{H}, \mathrm{CH}_{2}\right) ; 5.20(\mathrm{~s}, 1 \mathrm{H}$, $\mathrm{NH}) ; 7.25-7.40\left(\mathrm{~m}, 5 \mathrm{H}, \mathrm{ArH}^{\prime \prime}\right) ; 7.45-7.55(\mathrm{~m}, 3 \mathrm{H}$, ArH C3',4',5'); $8.18(\mathrm{~d}, \mathrm{~J}=9.0 \mathrm{~Hz}, 2 \mathrm{H}$, ArH C2',6'); 8.47 (s, 1H, C6-H) ppm; ${ }^{13} \mathrm{C}$ NMR (75 MHz, $\left.\mathrm{CDCl}_{3}\right): \delta=14.73\left(\mathrm{q}, 1 \mathrm{C}, \mathrm{CH}_{3}\right)$; 35.33 (t, 1C, $\left.\mathrm{CH}_{2}\right) ; 41.70\left(\mathrm{t}, 1 \mathrm{C}, \mathrm{CH}_{2}\right) ; 101.33$ (s, 1C, $\operatorname{ArC~C~3a);~} 121.38$ (d, 2C, ArC C 2',6'); 126.14 (d, 2C,ArC C4', C4"); 126.89 (d, 2C, ArC C2",6"); 128.89 (d, 2C, ArC C3",5"); 129.08 (d, 2C, ArC C3',5'); 138.56 (s,1C, ArC C 1'); 138.89 (s, 1C, ArC C 1'); 141.17 (s, 1C, ArC C 3); 153.99 (s, 1C, ArC C7a); 156.66 (s, 1C, ArC C6); 157.53 (s, 1C, ArC C4) ppm; IR (cm ${ }^{-1}$ ): $3428(\mathrm{NH}), 3030$ (CH aromatic), 2940, 2922 (CH aliphatic). MS (70 ev): $\mathrm{m} / \mathrm{z} 329\left(\mathrm{M}^{+}, 16.33 \%\right)$. Anal. Calcd for $\mathrm{C}_{20} \mathrm{H}_{19} \mathrm{~N}_{5}$ (329.40): C, 72.93; $\mathrm{H}, 5.81 ; \mathrm{N}, 21.26$.

Found: C, 72.73; H, 5.90; N, 21.11.

\section{3-Methyl-1-(4-nitrophenyl)-N-phenyl-1H-pyrazolo[3,4-d]pyrimidin-4-amine (Vd)}

Yield: $0.28 \mathrm{~g}(80 \%)$, M.p.: $259-260^{\circ} \mathrm{C}$ (benzene); ${ }^{1} \mathrm{H}$ NMR $(300 \mathrm{MHz}$, DMSO-d 6 ): $\delta=2.78$ (s, 3H, $\mathrm{CH}_{3}$ ); 7.20 (t, J = 7.2 Hz, 1H, ArH C4"); 7.40 (t, J = 7.8 Hz, 2H, ArH C3",5"); 7.68 (d, $\mathrm{J}=6.6 \mathrm{~Hz}, 2 \mathrm{H}$, ArH C2",6"); 8.40 (d, J = 7.2 Hz, 2H, ArH C2',6'); 8.49 (s, 1H, C6-H); 8.58 (d, $\mathrm{J}=7.2 \mathrm{~Hz}, 2 \mathrm{H}$, ArH C3',5'); $8.88\left(\mathrm{~s}, 1 \mathrm{H}, \mathrm{NH}, \mathrm{D} 2 \mathrm{O}\right.$ exchangeable) ppm; IR $\left(\mathrm{cm}^{-1}\right)$ : 3431 $(\mathrm{NH}), 3040$ ( $\mathrm{CH}$ aromatic), 2900 ( $\mathrm{CH}$ aliphatic), 1443, $1344\left(\mathrm{NO}_{2}\right)$. Anal. Calcd for $\mathrm{C}_{18} \mathrm{H}_{14} \mathrm{~N}_{6} \mathrm{O}_{2}$ (346.34): C, 62.42; H, 4.07; N, 24.27. Found: C, 62.59; H, 3.99; N, 24.57.

\section{3-Methyl-1-(4-nitrophenyl)-N-(2-phenylethyl)-1H-pyrazolo[3,4-d]pyrimidin-4-amine (Ve)}

Yield: $0.13 \mathrm{~g}(36 \%)$, M.p.: $214^{\circ} \mathrm{C}$ (benzene); ${ }^{1} \mathrm{H}$ NMR $\left(200 \mathrm{MHz}, \mathrm{DMSO}-\mathrm{d}_{6}\right): \delta=2.63$ (s, $\left.3 \mathrm{H}, \mathrm{CH}_{3}\right) ; 2.96\left(\mathrm{t}, \mathrm{J}=7.0 \mathrm{~Hz}, 2 \mathrm{H}, \mathrm{CH}_{2}\right) ; 3.80\left(\mathrm{t}, \mathrm{J}=7.0 \mathrm{~Hz}, 2 \mathrm{H}, \mathrm{CH}_{2}\right) ; 7.20-7.39(\mathrm{~m}, 5 \mathrm{H}$, 
ArH"); 7.53 (s, 1H, NH, D2O exchangeable); 8.39 (d, J = 9.4 Hz, 2H, ArH C3',5'); 8.45 (s, $1 \mathrm{H}, \mathrm{C} 6-\mathrm{H})$; 8.57 (d, J = $9.4 \mathrm{~Hz}, 2 \mathrm{H}$, ArH C2',6') ppm; IR $\left(\mathrm{cm}^{-1}\right)$ : $3439(\mathrm{NH}), 3080,30210$ (CH aromatic), 2940, 2920 ( $\mathrm{CH}$ aliphatic), 1441, $1346\left(\mathrm{NO}_{2}\right)$; $\mathrm{MS}$ (70 ev): m/z $374\left(\mathrm{M}^{+}\right.$, 13.47\%). Anal. Calcd for $\mathrm{C}_{20} \mathrm{H}_{18} \mathrm{~N}_{6} \mathrm{O}_{2}$ (374.4): C, 64.16; $\mathrm{H}, 4.85 ; \mathrm{N}, 22.45$. Found: $\mathrm{C}$, 64.27; H, 4.89; N, 22.66.

1-(4-Chlorophenyl)-N-(4-methoxyphenyl)-3-methyl-1H-pyrazolo[3,4-d]pyrimidin-4-amine (Vf)

Yield: $0.26 \mathrm{~g}(70 \%)$, M.p.: $198-200^{\circ} \mathrm{C}$ (ethanol); ${ }^{1} \mathrm{H}$ NMR $\left(300 \mathrm{MHz}, \mathrm{DMSO}-\mathrm{d}_{6}\right): \delta=2.74$ (s, 3H, $\mathrm{CH}_{3}$ ); 3.78 (s, 3H, $\left.\mathrm{OCH}_{3}\right) ; 6.97$ (d, J = 7.2 Hz, 2H, ArH C3",5"); 7.53 (d, J = 7.0 Hz, 2H, ArH C2",6"); 7.58 (d, J = 6.6 Hz, 2H, ArH C3',5'); 8.25 (d, J = 6.9 Hz, 2H, ArH C2',6'); $8.36(\mathrm{~s}, 1 \mathrm{H}, \mathrm{C} 6-\mathrm{H}) ; 8.74\left(\mathrm{~s}, 1 \mathrm{H}, \mathrm{NH}, \mathrm{D}_{2} \mathrm{O}\right.$ exchangeable) ppm; IR $\left(\mathrm{cm}^{-1}\right): 3443(\mathrm{NH}), 3010$ (CH aromatic), 2934, 2915 (CH aliphatic); MS (70 ev): $\mathrm{m} / z 365$ ( $\left.\mathrm{M}^{+}, 24.61 \%\right)$. Anal. Calcd for $\mathrm{C}_{19} \mathrm{H}_{16} \mathrm{ClN}_{5} \mathrm{O}$ (365.82): C, 62.38; $\mathrm{H}, 4.41 ; \mathrm{N}, 19.14$. Found: $\mathrm{C}, 65.59 ; \mathrm{H}, 4.43 ; \mathrm{N}, 18.95$.

1-(4-Chlorophenyl)-3-methyl-N-(2-phenylethyl)-1H-pyrazolo[3,4-d]pyrimidin-4-amine (Vg)

Yield: $0.24 \mathrm{~g}(65 \%)$, M.p.: $123-125^{\circ} \mathrm{C}$ (ethanol/water); ${ }^{1} \mathrm{H}$ NMR $\left(300 \mathrm{MHz}, \mathrm{DMSO}-\mathrm{d}_{6}\right): \delta=$ $2.61\left(\mathrm{~s}, 3 \mathrm{H}, \mathrm{CH}_{3}\right) ; 2.95\left(\mathrm{t}, \mathrm{J}=7.8 \mathrm{~Hz}, 2 \mathrm{H}, \mathrm{CH}_{2}\right) ; 3.76\left(\mathrm{t}, \mathrm{J}=7.5 \mathrm{~Hz}, 2 \mathrm{H}, \mathrm{CH}_{2}\right) ; 7.21-7.34(\mathrm{~m}$, $5 \mathrm{H}, \mathrm{ArH}$ "); 7.34 (s, 1H, NH, $\mathrm{D}_{2} \mathrm{O}$ exchangeable); 7.56 (d, J = 9.0 Hz, 2H, ArH C3',5'); 8.24 $\left(\mathrm{d}, \mathrm{J}=9.0 \mathrm{~Hz}, 2 \mathrm{H}, \mathrm{ArH} \mathrm{C2}, 6^{\prime}\right) ; 8.39(\mathrm{~s}, 1 \mathrm{H}, \mathrm{C} 6-\mathrm{H}) \mathrm{ppm}$; IR $\left(\mathrm{cm}^{-1}\right)$ : $3418(\mathrm{NH}), 3100,3026$ (CH aromatic), 2924, 2860 ( $\mathrm{CH}$ aliphatic). Anal. Calcd for $\mathrm{C}_{20} \mathrm{H}_{18} \mathrm{CIN}_{5}$ (363.84): C, 66.02; H, 4.99; N, 19.25. Found: C, 66.13; H, 5.15; N, 19.19.

\section{Synthesis of 1-(4-chlorophenyl)-4-hydrazino-3-methyl-1H-pyrazolo[3,4-d]pyrimidine (VI)}

Hydrazine hydrate $(0.1 \mathrm{~mol})$ was added to a suspension of IVc $(0.01 \mathrm{~mol})$ in ethanol $(35 \mathrm{ml})$, the reaction mixture was heated under reflux for $2.5 \mathrm{~h}$; the precipitated product was filtered, washed with ethanol, dried, and crystallized from ethanol.

Yield: $2.55 \mathrm{~g}$ (93\%), M.p.: $244-246^{\circ} \mathrm{C} ;{ }^{1} \mathrm{H}$ NMR (300 MHz, DMSO-d $\left.\mathrm{d}_{6}\right): \delta=2.62(\mathrm{~s}, 3 \mathrm{H}$, $\mathrm{CH}_{3}$ ); $3.82\left(\mathrm{~s}, 1 \mathrm{H}, \mathrm{NH}, \mathrm{D}_{2} \mathrm{O}\right.$ exchangeable); 4.95 (broad $\mathrm{s}, 2 \mathrm{H}, \mathrm{NH}_{2}, \mathrm{D}_{2} \mathrm{O}$ exchangeable); 7.56 (d, J = 9.0 Hz, 2H, H3',5'); 8.22 (d, J = 9.0 Hz, 2H, H2',6'); 8.36 (s, 1H, H6) ppm; IR $\left(\mathrm{cm}^{-1}\right): 3294(\mathrm{NH}), 3278$ \& $3199\left(\mathrm{NH}_{2}\right) ; \mathrm{MS}(70 \mathrm{ev}): \mathrm{m} / \mathrm{z} 276\left(\mathrm{M}^{+}+2\right) ; 274(100 \%)\left(\mathrm{M}^{+}\right)$. Anal. Calcd for $\mathrm{C}_{12} \mathrm{H}_{11} \mathrm{ClN}_{6}$ (274.71): C, 52.47; $\mathrm{H}, 4.04 ; \mathrm{N}, 30.59$. Found: $\mathrm{C}, 52.67 ; \mathrm{H}, 4.24$; N, 30.59.

\section{General procedure for the synthesis of compounds VIla-c}

A suspension of $\mathbf{V I}(1 \mathrm{mmol})$, and the appropriate aldehyde $(1 \mathrm{mmol})$ in ethanol $(35 \mathrm{ml})$, was heated under reflux with stirring for 3-4 h; the reaction mixture was then cooled and the separated precipitate was filtered, dried, and crystallized from ethanol.

2-(\{2-[1-(4-Chlorophenyl)-3-methyl-1H-pyrazolo[3,4-d]pyrimidin-4-yl]hydrazinylidene\}methyl)phenol (VIIa)

Yield: $0.24 \mathrm{~g}(63 \%)$, M.p.: $269-270^{\circ} \mathrm{C} ;{ }^{1} \mathrm{H}$ NMR (300 MHz, DMSO-d 6 ): $\delta=2.76(\mathrm{~s}, 3 \mathrm{H}$, $\left.\mathrm{CH}_{3}\right) ; 6.88-6.95$ (m, 1H, H5"); 7.28-7.33 (m, 1H, H3"); 7.39-7.43 (m, 1H, H4"); 7.58-7.63 $(\mathrm{m}, 1 \mathrm{H}, \mathrm{H6}$ "); 7.80 (d, J = 9.0 Hz, 2H, H3',5'); 8.04 (d, J = 9.0 Hz, 2H, H2',6'); 8.23 (s, 1H, $\mathrm{HC}=\mathrm{N}) ; 8.60(\mathrm{~s}, 1 \mathrm{H}, \mathrm{H} 6) ; 10.20$ (broad s, $1 \mathrm{H}, \mathrm{OH}, \mathrm{D}_{2} \mathrm{O}$ exchangeable); 11.90 (broad s, $1 \mathrm{H}$, 
$\mathrm{NH}, \mathrm{D}_{2} \mathrm{O}$ exchangeable) ppm; ${ }^{13} \mathrm{C}$ NMR $\left(75 \mathrm{MHz}, \mathrm{DMSO}-\mathrm{d}_{6}\right): \delta=14.10$ (q, $1 \mathrm{C}, \mathrm{CH}_{3}$ ); 102.00 (s, 1C, ArC C3a); 117.80 (s,1C, ArC C 3"); 119.80 (d, 2C, ArC C2',6'); 125.38 (s,1C, ArC C5"); 127.80 (s,1C, ArC C6"); 129.00 (d,2C, ArC C3',5'); 131.50 (s,1C, ArC C4'); 132.90 (s,1C, ArC C 4"); 136.54 (s, 1C, ArC C 1"); 138.26 (s,1C, ArC C 1'); 144.93 (s, 1C,C=N); 147.85 (s, 1C, ArC C3); 149.50 (s, 1C, ArC C7a); 153.57 (s, 1C, ArC C6); 157.47 (s, 1C, $\operatorname{ArC} C 2 ") ; 157.71$ (s, 1C, ArC C4) ppm; IR (cm $\left.{ }^{-1}\right): 3433(\mathrm{NH}), 3369(\mathrm{OH})$; MS (70 ev): $\mathrm{m} / \mathrm{z} 380\left(\mathrm{M}^{+}+2\right) ; 378\left(\mathrm{M}^{+}\right) ; 243(100 \%)$. Anal. Calcd for $\mathrm{C}_{19} \mathrm{H}_{15} \mathrm{ClN}_{6} \mathrm{O}$ (378.82): C, 60.24; H, 3.99; N, 22.19. Found: C, 60.50; H, 3.89; N, 22.44.

1-(4-Chlorophenyl)-4-[2-(4-methoxybenzylidene)hydrazinyl]-3-methyl-1Hpyrazolo[3,4-d]pyrimidine (VIIb)

Yield: $0.32 \mathrm{~g}$ (82\%), M.p.: $247-248^{\circ} \mathrm{C} ;{ }^{1} \mathrm{H}$ NMR (300 MHz, DMSO-d 6 ): $\delta=2.79$ (s, $3 \mathrm{H}$, $\mathrm{CH}_{3}$ ); $3.82\left(\mathrm{~s}, 3 \mathrm{H}, \mathrm{OCH}_{3}\right) ; 7.00$ (d, J = 8.7 Hz, 2H, H3",5"); 7.60 (d, J = 9.0 Hz, 2H, H3',5'); 7.65 (d, J = 8.7 Hz, 2H, H2",6"); 7.95 (d, J = 9.0 Hz, 2H, H2',6'); 8.20 (s, 1H, HC=N); 8.35 (s, $1 \mathrm{H}, \mathrm{H} 6$ ); 11.87 (broad s, $1 \mathrm{H}, \mathrm{NH}, \mathrm{D}_{2} \mathrm{O}$ exchangeable) ppm; IR (cm $\left.{ }^{-1}\right): 3220(\mathrm{NH})$; MS (70 ev): $\mathrm{m} / \mathrm{z} 393\left(\mathrm{M}^{+}+1\right)$; $392(100 \%)$. Anal. Calcd for $\mathrm{C}_{20} \mathrm{H}_{17} \mathrm{CIN}_{6} \mathrm{O}$ (392.84): C, 61.15; $\mathrm{H}$, 4.36; N, 21.39. Found: C, 61.39; H, 4.51; N, 21.10.

1-(4-Chlorophenyl)-3-methyl-4-[2-(2-nitrobenzylidene)hydrazinyl]-1Hpyrazolo[3,4-d]pyrimidine (VIIc)

Yield: $0.35 \mathrm{~g}$ (86\%), M.p.: $286-288^{\circ} \mathrm{C} ;{ }^{1} \mathrm{H}$ NMR (300 MHz, DMSO-d $\left.\mathrm{d}_{6}\right): \delta=2.77$ (s, $3 \mathrm{H}$, $\mathrm{CH}_{3}$ ); 7.56 (d, J = 9.0 Hz, 2H, H3',5'); $7.78-7.84\left(\mathrm{~m}, 2 \mathrm{H}, \mathrm{H} 5^{\prime \prime}, 6\right.$ "); 8.07 (d, J = 9.0 Hz, 2H, $\left.\mathrm{H}^{\prime}, 6^{\prime}\right)$; 8.08-8.13 (m, 1H, H4"); 8.33 (s, 1H, HC=N); 8.53 (s, 1H, H6); 8.75-8.80 (m, 1H, $\mathrm{H} 2$ "); 12.12 (broad s, $1 \mathrm{H}, \mathrm{NH}, \mathrm{D}_{2} \mathrm{O}$ exchangeable) ppm; IR $\left(\mathrm{cm}^{-1}\right)$ : $3327(\mathrm{NH}), 1437,1346$ $\left(\mathrm{NO}_{2}\right)$. Anal. Calcd for $\mathrm{C}_{19} \mathrm{H}_{14} \mathrm{ClN}_{7} \mathrm{O}_{2}$ (407.81): C, 55.96; $\mathrm{H}, 3.46 ; \mathrm{N}, 24.04$. Found: C, 56.20; H, 3.56; N, 23.96.

\section{Antitumor activity}

The compounds were prepared in DMSO: glycerol 9:1 at a concentration of $4 \mathrm{mM}$ for the single-dose assay. The solution was diluted 1:400, giving a test concentration of $10 \mu \mathrm{M}$. The human tumor cell lines of the cancer screening panel were prepared according to the standard procedure of the American National Cancer Institute $(\mathrm{NCl})$, and the tests were performed at the American National Cancer Institute ( $\mathrm{NCl})[23,24]$.

Compound VIla, which was subjected to a 5-dose assay, was prepared at a concentration $40 \mathrm{mM}$. The solution was diluted 1:400, giving a Test concentration of $100 \mu \mathrm{M}$.

The human tumor cell lines of the cancer screening panel were prepared according to the standard procedures of the $\mathrm{NCl}$. The results are summarized in Tables 1 and 2, and Figure 2. 


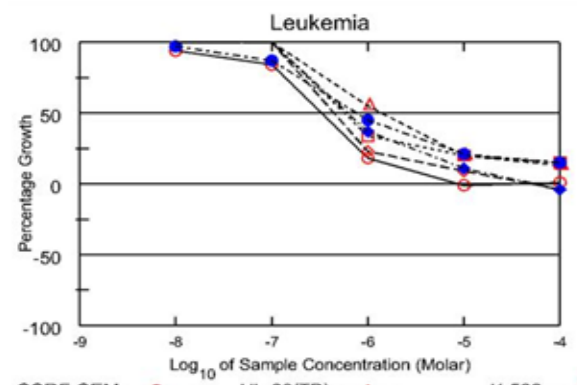

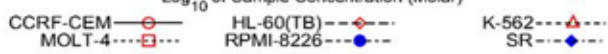
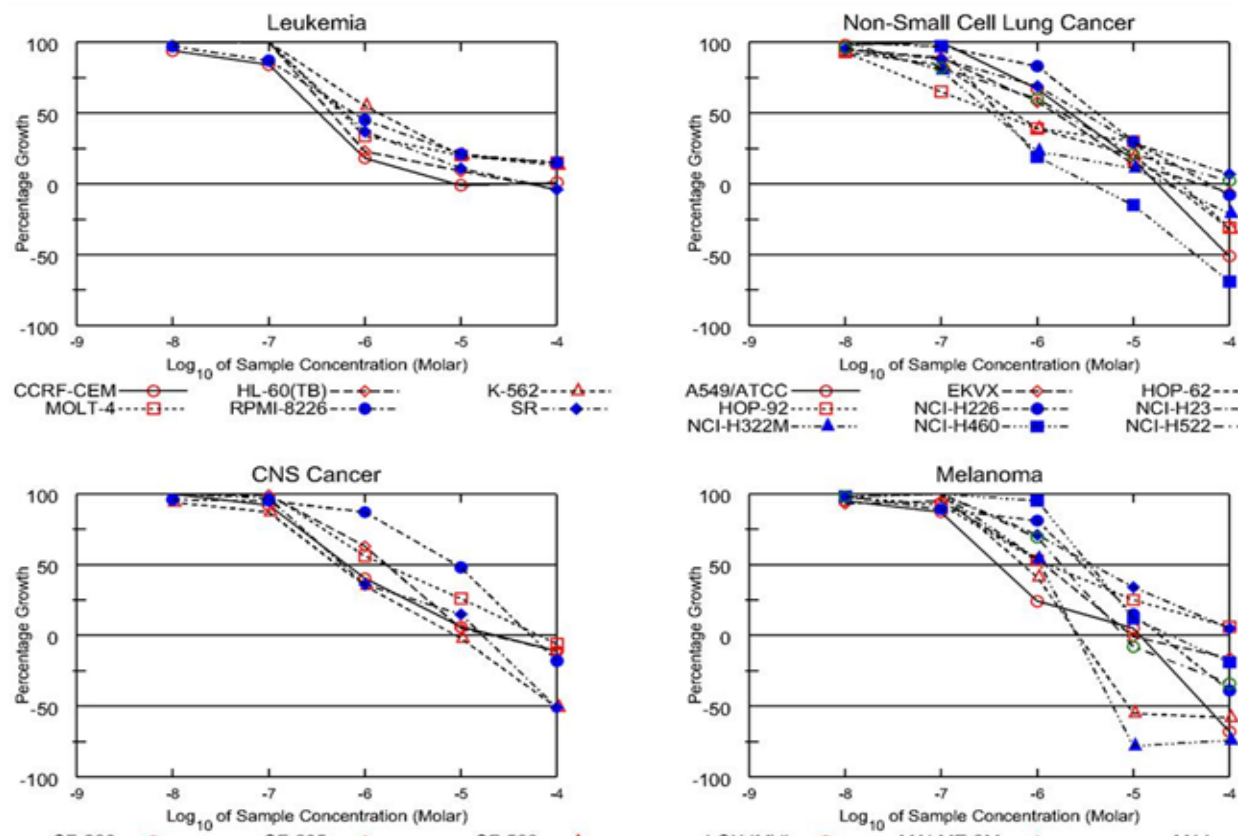

SF-268- $\log _{10}$ of Sample Concentration (Molar)
SNB-19.......

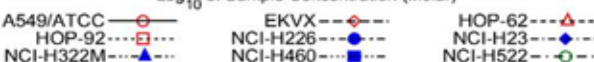

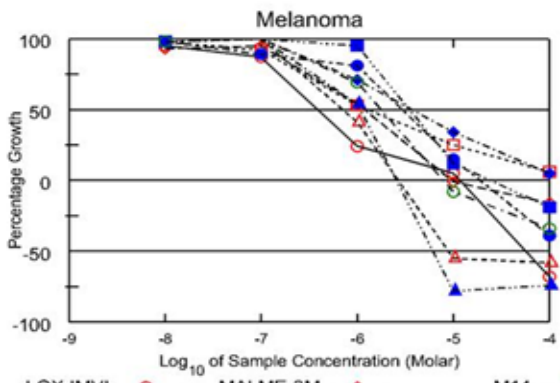

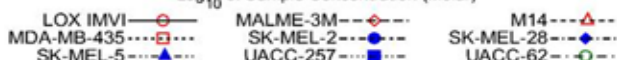

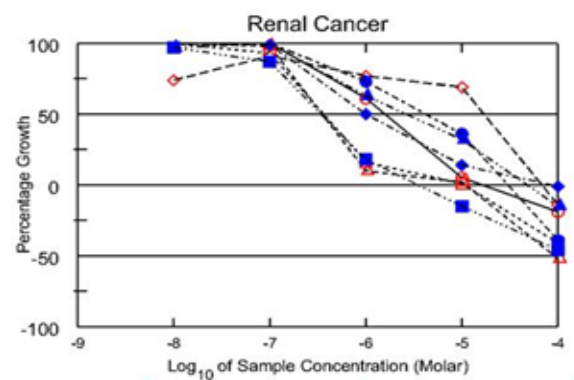
CAKI-1....
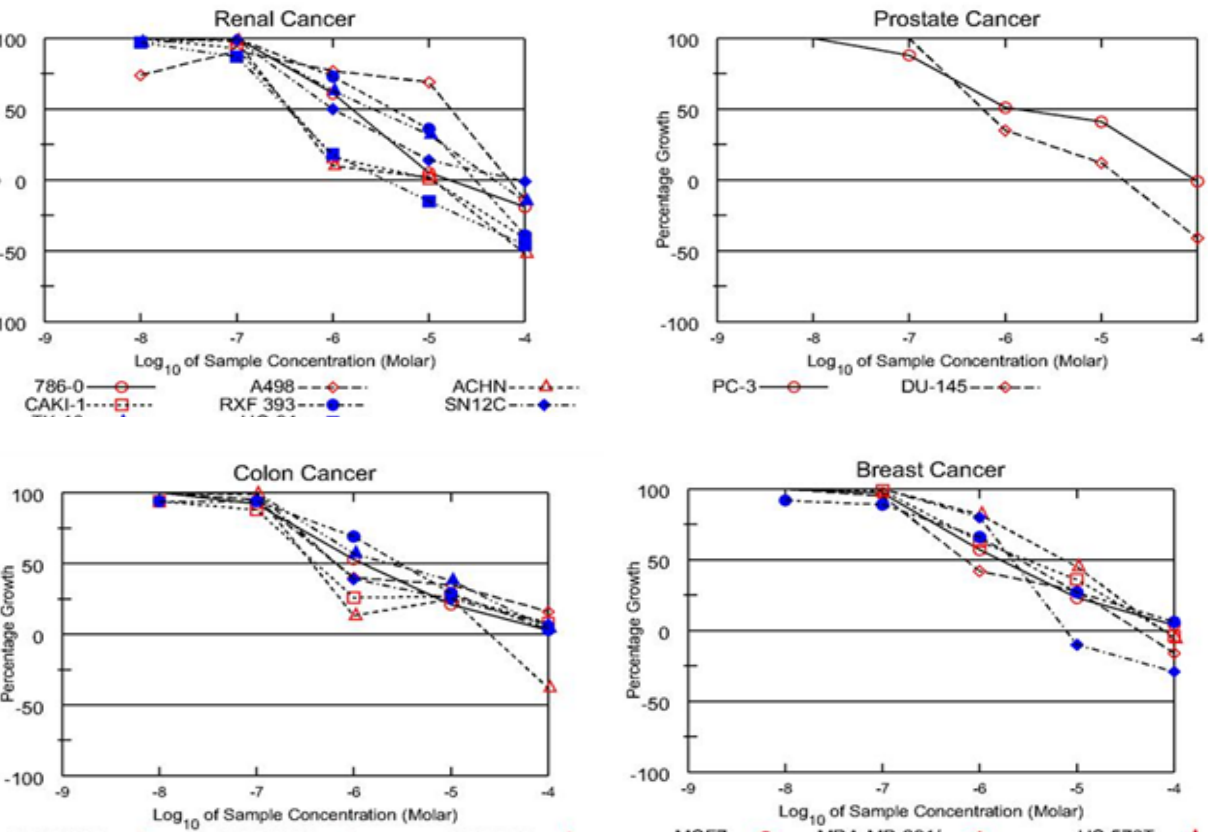

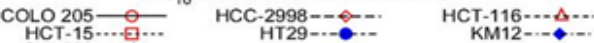

HCT-15…… HT29-...

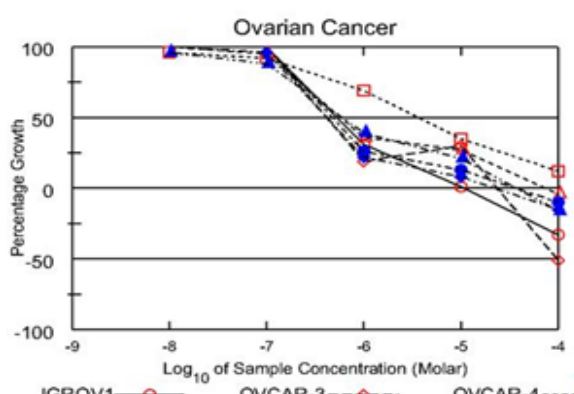

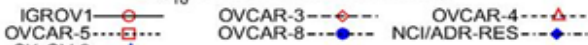

Fig. 2. Dose response curves for compound Vlla. 


\section{Conclusion}

The objective of this study was to synthesize and investigate the anticancer inhibition activity of selected pyrazolopyrimidines with the hope of discovering new structure leads to serve as potential anticancer agents. The newly synthesized compounds showed good inhibitory activity on different cell lines at a concentration of $10 \mu \mathrm{M}$, making them leading chemical entities for further modification to render them as clinically useful therapeutic agents. Compound Vc showed $65.57 \%$ and $53.25 \%$ on the k-562 and molt-4 cell lines of leukemia. Vg produced 76.32 and 51.72 percentage inhibition on the K-562 and HOP-92 cell lines of leukemia and small lung cancer, VIlc showed $90 \%$ inhibition on the ovcar-3 cell line of ovarian cancer, and $82 \%$ and $84 \%$ inhibition on the k-562 and molt-4 cell lines of leukemia. The highest activity was presented by compound VIla, which showed good inhibitory activity against 57 cell lines, and was the reason for performing 5-dose testing on this compound and measuring its GI50 (growth inhibition)and LC50 (lethal concentration), which further revealed its high potency against most of the cell lines.

\section{Acknowledgement}

We would like to thank the American National Cancer Institute $(\mathrm{NCl})$ team for their help in carrying out the antitumor screening of the newly synthesized compounds.

\section{Authors' Statement}

\section{Competing Interests}

The authors declare no conflict of interest.

\section{References}

[1] Zhang X, Lin Q, Zhong P.

A Facile One-pot Synthesis of 1-Arylpyrazolo[3,4-d]pyrimidin-4-ones.

Molecules. 2010; 15: 3079-3086.

http://dx.doi.org/10.3390/molecules15053079

[2] Kim DC, Lee YR, Yang B, Shin KJ, Kim DJ, Chung BY, Yoo KH.

Synthesis and biological evaluations of pyrazolo[3,4-d]pyrimidines as cyclin-dependent kinase 2 inhibitors.

Eur J Med Chem. 2003; 38: 525-532.

http://dx.doi.org/10.1016/S0223-5234(03)00065-5

[3] Markwalder JA, Arnone MR, Benfield PA, Boisclair M, Burton CR, Chang C, Cox SS, Czerniak PM, Dean CL, Doleniak D, Grafstrom R, Harrison BA, Kaltenbach RF, Nugiel DA, Rossi KA, Sherk SR, Sisk LM, Stouten P, Trainor GL, Worland P, Seitz SP.

Synthesis and Biological Evaluation of 1-Aryl-4,5-dihydro-1H-pyrazolo[3,4-d]pyrimidin-4-one Inhibitors of Cyclin-Dependent Kinases.

J Med Chem. 2004; 47: 5894-5911.

http://dx.doi.org/10.1021/jm020455u

[4] Ibrahim DA, El-Metwally AM, Al-Arab EE.

Structure-based design of a new class of highly selective pyrazolo[3,4-d]pyrimidines based inhibitors of cyclin dependent kinases.

Arkivoc. 2009; 7: 12-25. 
[5] Schenone S, Brullo C, Bruno O, Bondavalli F, Mosti L, Maga G, Crespan E, Carraro F, Manetti F, Tintori C, Botta M.

Synthesis, biological evaluation and docking studies of 4-amino substituted

$1 \mathrm{H}$-pyrazolo[3,4-d]pyrimidines.

Eur J Med Chem. 2008; 43: 2665-2676.

http://dx.doi.org/10.1016/j.ejmech.2008.01.034

[6] Peat AJ, Boucheron JA, Dickerson SH, Garrido D, Mills W, Peckham J, Preugschat F, Smalley T, Schweiker SL, Wilson JR, Wang TY, Zhou HQ, Thomson SA.

Novel pyrazolopyrimidine derivatives as GSK-3 inhibitors.

Bioorg Med Chem Lett. 2004; 14: 2121-2125.

http://dx.doi.org/10.1016/j.bmcl.2004.02.036

[7] Smalley TL, Peat AJ, Boucheron JA, Dickerson S, Garrido D, Preugschat F, Schweiker SL, Thomson SA, Wang TY.

Synthesis and evaluation of novel heterocyclic inhibitors of GSK-3.

Bioorg Med Chem Lett. 2006; 16: 2091-2094.

http://dx.doi.org/10.1016/j.bmcl.2006.01.057

[8] Dessalew N, Patel DS, Bharatam PV.

3D-QSAR and molecular docking studies on pyrazolopyrimidine derivatives as glycogen synthase kinase-3 inhibitors.

J Mol Graphics Modell. 2007; 25: 885-895.

http://dx.doi.org/10.1016/j.jmgm.2006.08.009

[9] Settimo FD, Primofiore G, Motta CL, Taliani S, Simorini F, Marini AM, Mugnaini L, Lavecchia A, Novellino E, Tuscano D, Martini C.

Novel, Highly Potent Adenosine Deaminase Inhibitors Containing the Pyrazolo[3,4-d]pyrimidine Ring System. Synthesis, Structure-Activity Relationships, and Molecular Modeling Studies.

J Med Chem. 2005; 48: 5162-5174.

http://dx.doi.org/10.1021/jm050136d

[10] Hubbard RD, Bamaung NY, Palazzo F, Zhang Q, Kovar P, Osterling DJ, Hu X, Wilsbacher JL, Johnson EF, Bouska J, Wang J, Bell RL, Davidsen SK, Sheppard GS.

Pyrazolo[3,4-d]pyrimidines as potent inhibitors of the insulin-like growth factor receptor (IGF-IR). Bioorg Med Chem Lett. 2007; 17: 5406-5409.

http://dx.doi.org/10.1016/j.bmcl.2007.07.037

[11] Gupta S, Rodrigues LM, Esteves AP, Oliveira-Campos AMF, Nascimento MSJ, Nazareth N, Cidade H, Neves MP, Fernandes E, Pinto M, Cerqueira NMFSA, Bras N.

Synthesis of $\mathrm{N}$-aryl-5-amino-4-cyanopyrazole derivatives as potent xanthine oxidase inhibitors.

Eur J Med Chem. 2008; 43: 771-780.

http://dx.doi.org/10.1016/j.ejmech.2007.06.002

[12] Abdelrazek HA, Abdelwahab AE.

Synthesis and Biological Evaluation of Some Novel Fused Pyrazolopyrimidines as Potential

Anticancer and Antimicrobial Agents.

Arch Pharm Chem Life Sci. 2011; 11: 184-196.

http://dx.doi.org/10.1002/ardp.201000188

[13] Schenone S, Bruno O, Bondavalli F, Ranise A, Mosti L, Menozzi G, Fossa P, Donnini S, Santoro A, Ziche M, Manetti F, Botta M.

1-Aryl-4-amino-1H-pyrazolo[3,4-d]pyrimidine derivatives toward the human epidermoid carcinomaA431 cell line.

Eur J Med Chem. 2004; 39: 939-946.

http://dx.doi.org/10.1016/j.ejmech.2004.07.010

[14] Ghorab MM, Ragab FA, Alqasoumi SI, Alafeefy AM, Aboulmagd SA.

Synthesis of some new pyrazolo[3,4-d]pyrimidine derivatives of expected anticancer and radioprotective activity.

Eur J Med Chem. 2010; 45: 171-178.

http://dx.doi.org/10.1016/j.ejmech.2009.09.039 
[15] Curran KJ, Verheijen JC, Kaplan J, Richard DJ, Toral-Barza L, Hollander I, Lucas J, Ayral-Kaloustian S, Yu K, Zask A.

Pyrazolopyrimidines as highly potent and selective, ATP-competitive inhibitors of the mammalian target of rapamycin (mTOR): Optimization of the 1-substituent.

Bioorg Med Chem Lett. 2010; 20: 1440-1444.

http://dx.doi.org/10.1016/j.bmcl.2009.12.086

[16] Li J, Zhu Y, Wang X, Yang $\mathrm{H}$.

Synthesis and Herbicidal Activities of a Series of Di(aminopyrazoly) Ketone Derivatives.

J Heterocyclic Chem. 2007; 44: 749-755.

http://dx.doi.org/10.1002/jhet.5570440401

[17] O'Halloran N, James JP, Downey CA, O'Malley P, Duff T, Bertrand S.

Inter- and intra- molecular cyclisation reactions of azoacetates derived from aryl hydrazones of ethyl acetoacetate and acetoacetanilides.

Heterocycles. 2008; 75: 2681-2701.

http://dx.doi.org/10.3987/COM-08-11433

[18] Cheng CC, Robins RK.

Potential Purine Antagonists. VI. Synthesis of 1-Alkyl- and 1-Aryl-4-substituted

Pyrazolo[3,4-d]pyrimidine.

J Org Chem. 1956; 21: 1240-1256.

http://dx.doi.org/10.1021/jo01117a010

[19] Kreutzberger A, Burgwitz K.

Antimycotic drugs. XI: 4-Amino-3-methylpyrazolo[3,4-d]pyrimidines.

Arch Pharm. 1980; 313; 906-912.

http://dx.doi.org/10.1002/ardp.19803131103

[20] Silvestri R, Cascio MG, Regina GL, Piscitelli F, Lavecchia A, Brizzi A, Pasquini S, Botta M, Novellino E, Marzo VD, Corelli F.

Synthesis, Cannabinoid Receptor Affinity, and Molecular Modeling Studies of Substituted

1-Aryl-5-(1H-pyrrol-1-yl)-1H-pyrazole-3-carboxamides.

J Med Chem. 2008; 51: 1560-1576.

http://dx.doi.org/10.1021/jm070566z

[21] Davoodnia A, Rahimizadeh M, Rivadeh S, Bakavoli M, Roshani M.

Synthesis of new substituted pyrazolo[3,4-d]pyrimidin-4-ones under microwave irradiation. Indian J Heterocycl Chem. 2006; 16: 151-154.

[22] Davoodnia A, Zhiani R, Tavakoli-Hoseini N.

Synthesis of pyrazolo[4,3-e][1,2,4]triazolo[4,3-c]pyrimidines.

Monatsh Chem. 2008; 139: 1405-1407.

http://dx.doi.org/10.1007/s00706-008-0939-8

[23] Boyd MR, Paull KD.

Some Practical Considerations and Applications of the National Cancer Institute In-Vitro Anticancer

Drug Discovery Screen.

Drug Dev Res. 1995; 34: 91-109.

http://dx.doi.org/10.1002/ddr.430340203

[24] Skehan P, Storeng R, Scudiero D, Monks A, McMahon J, Vistica D, Warren J R, Bokesch H, Kenney S, Boyd MR.

New colorimetric cytotoxic assay for anticancer drug screening.

J Natl Cancer Inst. 1990; 82: 1107-1112.

http://dx.doi.org/10.1093/jnci/82.13.1107 